\title{
Polymer Microring Resonators for High-Frequency Ultrasound Detection and Imaging
}

\author{
Adam Maxwell, Sheng-Wen Huang, Member, IEEE, Tao Ling, Jin-Sung Kim, \\ Shai Ashkenazi, Member, IEEE, and L. Jay Guo, Member, IEEE
}

\begin{abstract}
Polymer microring resonators fabricated by nanoimprinting are presented as a means of ultrasound detection. Acoustic waves impinging on a ring-shaped optical resonator cause strain in the ring dimensions, modulating optical output. Basic acoustic and optical characteristics of the microring sensor are presented. Measurements at several frequencies show a high sensitivity and low noise-equivalent pressure. The angular response is determined by sensing the optoacoustic excitation of a $49 \mu \mathrm{m}$ polyester microsphere and shows wide-angle sensitivity. A 1-D array consisting of four microrings is demonstrated using wavelength multiplexing for addressing each element. The high sensitivity, bandwidth, and angular response make it a potentially useful sensor platform for many applications including high-frequency ultrasonic and photoacoustic imaging.
\end{abstract}

Index Terms-Acoustic detectors, acoustic devices, microresonators, optical resonance, optical resonators, optical strip waveguides, optical waveguides, optical waveguide components, ultrasound detector.

\section{INTRODUCTION}

$\mathbf{H}$ IGH-FREQUENCY ( $>50 \mathrm{MHz}$ ) ultrasound imaging is an excellent tool for small animal studies and in vitro biological microscopy [1] providing high-resolution real-time imaging. However, its clinical use is limited (primarily to ocular imaging) due to technology limitations on element size, spacing, and signal transduction in traditional ultrasound imaging. The optical detection of ultrasound is an alternative to the traditional piezoelectric technology with unique advantages for high-frequency arrays.

1) Element size in the range of $10-100 \mu \mathrm{m}$ can be realized using optical microresonators with sufficient sensitivity.

2) Unlike piezoelectric detectors, the noise in optical detection does not directly depend on the detector size. Moreover, the SNR can be increased by increasing the power of the input optical probing.

Manuscript received September 6, 2007; revised November 20, 2007. This work was supported by the NIH under Grant EB004933-01 and Grant EB007619-01A1.

A. Maxwell, T. Ling, and L. J. Guo are with the Department of Electrical Engineering and Computer Science, University of Michigan, Ann Arbor, MI 48109 USA (e-mail: guo@eecs.umich.edu).

S.-W. Huang and S. Ashkenazi are with the Department of Biomedical Engineering, University of Michigan, Ann Arbor, MI 48109 USA (e-mail: shaia@umich.edu).

J.-S. Kim was with the Department of Electrical Engineering and Computer Science, University of Michigan, Ann Arbor, MI 48109 USA. He is now with the Devices \& Materials Laboratory, LG Electronics Institute of Technology, Seoul 137-724, Korea.

Color versions of one or more of the figures in this paper are available online at http://ieeexplore.ieee.org.

Digital Object Identifier 10.1109/JSTQE.2007.914047

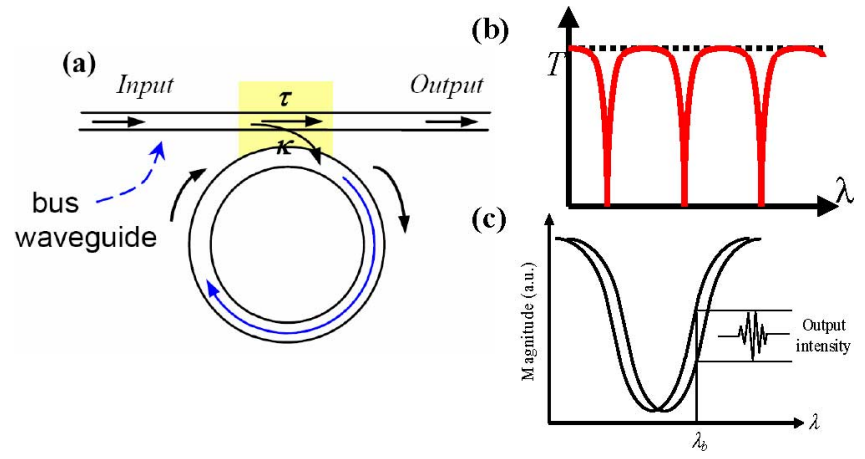

Fig. 1. Schematic of (a) microring resonator, (b) resonance spectrum, (c) ultrasound detection using the optical resonance of a microring sensor.

3) High element count of 1-D and 2-D array configurations can share a single optical fiber for signal interfacing using optical multiplexing.

These features can be exploited for submillimeter imaging devices optimized for intravascular imaging and in vivo microscopy or virtual biopsy using needle mounted probe.

In this paper, we present our study and development of a new planar optic platform for detecting ultrasound: polymer resonator for optical ultrasound detection (PROUD). We will start with a description of the theoretical background and principles for ultrasound sensing using optical microring resonators. Next, we will present our device fabrication methods and experimental characterization of the PROUD sensor and a four-element array. We will show that the polymer microring resonators are capable of high-frequency ultrasound detection with high sensitivity, and when constructed as an array, addressing of each detector element can be achieved by wavelength division multiplexing through a single input/output bus. Our experiment shows that the noise-equivalent pressure (NEP) of our present PROUD sensor is already comparable to the state-of-the-art hydrophone of similar size. To conclude, we will discuss the challenges in developing the technology, possible solutions, and typical applications.

\section{PrinciPles OF Microring Ultrasound SENSORS}

\section{A. Microring Resonators for Ultrasound Detection}

A microring resonator is composed of a ring waveguide closely coupled with a bus waveguide that serves as input/output, as shown in Fig. 1(a). Resonance condition occurs when the round-trip phase acquired by the guided wave in the microring is equal to $2 m \pi$, where $m$ is an integer. Under such condition, 
it can be shown that the field of the optical wave returning to the coupler after a round trip is exactly $\pi$ out of phase with the optical wave traveling through the coupler region in the straight waveguide. These two fields, therefore, interfere destructively, leading to a resonance dip in the transmission curve.

Microresonators can be used as detectors because their resonances can respond sensitively to the change in the effective refractive index $\left(n_{\mathrm{eff}}\right)$ of the guided optical mode [2]. It is this characteristic that is exploited in this paper for novel implementation of sensitive high-frequency ultrasound detectors. This aspect can be seen explicitly from the optical transmission through the bus waveguide coupled with a microring resonator, which can be expressed as

$$
T \equiv \frac{I_{t}}{I_{i}}=\alpha_{i}^{2} \frac{\left(\tau-\alpha_{i} a\right)^{2}+4 \tau \alpha_{i} a \sin ^{2} \frac{\phi}{2}}{\left(1-\tau \alpha_{i} a\right)^{2}+4 \tau \alpha_{i} a \sin ^{2} \frac{\phi}{2}}
$$

where $I_{t}$ and $I_{i}$ are incident and transmitted power, respectively, $\tau$ the amplitude transmission coefficient, $a$ the single-pass amplitude attenuation factor, and $\alpha_{\mathrm{i}}$ the insertion loss due to the waveguide mode mismatch in the coupling region. In (1), $\phi$ is the single-pass phase shift experienced by light traveling inside the microring, which is equal to $2 \pi n_{\mathrm{eff}} L / \lambda$. Here, $n_{\mathrm{eff}}$ is the effective refractive index of the mode guided inside the ring waveguide, $L$ the circumference of the ring, and $\lambda$ the light wavelength. The transmission of the system as expressed by (1) clearly has a strong dependence on the phase shift $\phi$, and therefore, is a function of the effective index. Based on our current understanding, the principle for the detection of ultrasound signal is that the acoustic pressure deforms the polymer waveguide and changes the effective index of the propagating mode. Additionally, there is a minor elastooptic effect that contributes to the effective index change. The theoretical understanding was described in detail in a recent publication [3]. In microresonator sensors, detections can be made by measuring the resonance shifts [4] or alternatively by measuring the output intensity changes from the device at a fixed wavelength [see Fig. 1(c)]. The latter scheme is especially useful for detecting very small change in the effective index, and our results show that such a property can be used to detect, sensitively, the microring response to an incident ultrasound pulse.

\section{DEVICE FABRICATION}

We used a nanoimprinting technique to fabricate the polymer microring resonators [5] that can be applied to many polymer materials [6]. In this technique, the waveguides are replicated by using a mold with inverse waveguide patterns defined on its surface to emboss into polymer material under controlled temperature and pressure. Very briefly, a mold is first fabricated by using a combination of electron-beam lithography, reactive ion etching (RIE) followed by a wet chemical etching to produce smooth sidewalls. Next, the ring resonator devices were prepared as follows: polystyrene (PS) of $350 \mathrm{~nm}$ thickness was spin coated on a 4- $\mu$ m-thick $\mathrm{SiO}_{2}$ layer thermally grown on a Si substrate, and prebaked at $180{ }^{\circ} \mathrm{C}$ to remove any residual solvent. We used Nanonex-2000 nanoimprinter (Nanonex, NJ) to transfer the microring resonator patterns from the mold onto
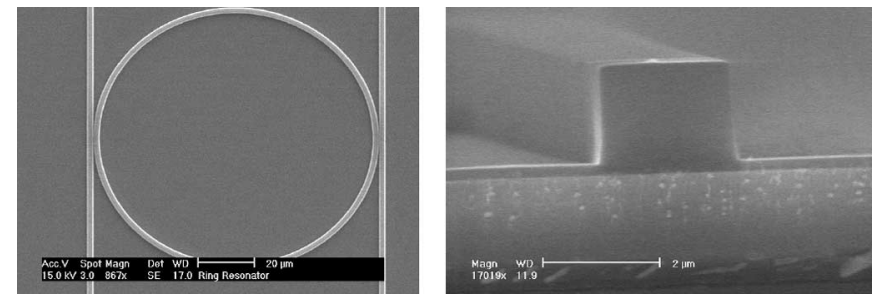

Fig. 2. Scanning electron micrographs of the PS microring resonators. (a) Top view (in this SEM, the microring is coupled to both a bus waveguide and a drop port waveguide). (b) Cross-section view of the PS waveguide.

the PS film. The sample stage was heated to $180^{\circ} \mathrm{C}$, and the PS resist was imprinted at a pressure of 600 psi for $300 \mathrm{~s}$. The sample stage was then cooled to room temperature by air, and the imprinting pressure was reduced to atmospheric pressure. After the sample was released from the mold, an $\mathrm{O}_{2}$ RIE process of $300 \mathrm{~s}$ was used to remove the residual layer, leaving the polymer waveguide on top of the $\mathrm{SiO}_{2}$ layer, which completed the device fabrication. In our fabrication, typically a large numbers of device structures are designed on the mold, which can imprint and create large arrays of polymer microring devices simultaneously. Fig. 2 show the scanning electron micrographs of the fabricated PS microring devices.

\section{EXPERIMENTAL PROUD CHARACTERISTICS}

\section{A. Experimental Setup}

The optical fibers were butt coupled to the input and output of the bus waveguide at the cleaved edges of the silicon substrate. The input fiber was connected to a continuous wave (CW) tunable laser source (HP 8168F, Agilent Technologies, Santa Clara, CA), and the output fiber was connected to a high-speed photodetector (1811-FC, New Focus, San Jose, CA) with a gain of $4 \times 10^{4} \mathrm{~V} / \mathrm{A}$ and an electrical bandwidth of dc $125 \mathrm{MHz}$. The photodetector was connected to a digital storage oscilloscope (WaveSurfer 432, LeCroy, Chestnut Ridge, NY) for data collection. A single-mode fiber was used for the input to maximize the amount of light coupled into the polymer waveguide. A multimode fiber $(62.5 \mu \mathrm{m}$ core diameter $)$ was aligned with the output of the waveguide. Given the dimensions of the input fiber ( $4 \mu \mathrm{m}$ core radius) and the polymer waveguide ( $2 \mu \mathrm{m} \times 2 \mu \mathrm{m})$, the maximum light that can be coupled into the waveguide is $8 \%$. This could be greatly improved by replacing the single-mode fiber with a tapered fiber with a tip diameter smaller than the waveguide dimensions. UV-curable epoxy was used to bond the fibers to the waveguide and minimize the reflection loss at the input and output. With a laser input power of $4 \mathrm{~mW}$, the maximum achievable power at the photodetector is $320 \mu \mathrm{W}$. However, due to scattering loss in the bus waveguide, the output power is only $20-30 \mu \mathrm{W}$.

The fiber-connected microring device (on a 500- $\mu$ m-thick silicon substrate) is mounted on a $200-\mu \mathrm{m}$-thick rigid plate and submerged in a tank filled with deionized water (see Fig. 3). Microrings used in the following experiments have a $100 \mu \mathrm{m}$ diameter. The spectrum is measured over several periods of resonance by sweeping the tunable laser wavelength. A suitable 


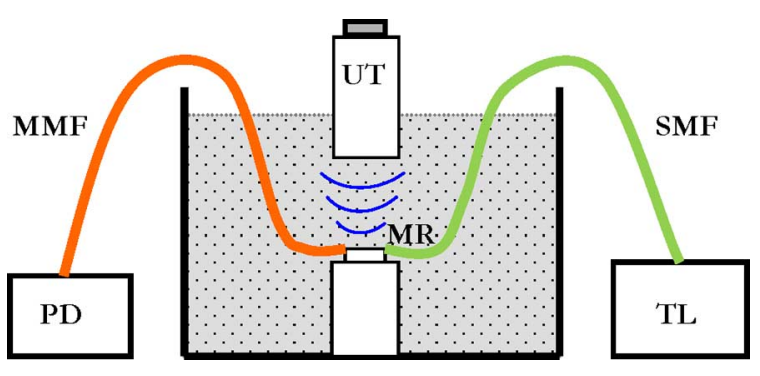

Fig. 3. Experiment setup used to determine the microring sensor characteristics. Light from a fiber-coupled tunable laser is coupled into the microring waveguide bus, and output to an amplified photodetector. The microring is positioned at the center of the unfocused ultrasound transducer. The distance between the microring and transducer is about $1 \mathrm{~mm}$.

wavelength near the center of the resonant slope is then chosen. The $Q$ factor of the current microrings is approximately $5 \times 10^{3}$.

\section{B. Sensitivity and NEP}

The sensitivity is determined by the slope of the resonance, while the dynamic range of the microring is determined by the depth of the resonance, i.e., the range of the linear region. If the shift in resonance is too great, the wavelength will be beyond the resonance resulting in signal distortion and decreased sensitivity. The NEP is defined as the ratio of sensitivity (in micowatt per megapascal) to the noise-equivalent optical power (in micowatt), and is a measure of the minimum detectable pressure of the sensor [7]. For the microring, this is dependant on the sensitivity and the optical output power.

In order to measure the dynamic sensitivity, a $20 \mathrm{MHz}$ unfocused transducer (V316, Panametrics NDT, Waltham, MA) with a $3.18 \mathrm{~mm}$ diameter was used to insonify the ring. The transducer was calibrated with a polyvinylidene fluoride (PVDF) membrane hydrophone with $28 \mu \mathrm{m}$ membrane thickness and a $500 \mu \mathrm{m}$ active element [8] (Center for Industrial and Medical Ultrasound, University of Washington, Seattle, WA). Signals from the membrane hydrophone and microring resonator were recorded with the transducer driven by a one-cycle $20 \mathrm{MHz}$ sinusoidal wave from a function generator (33250A, Agilent Technologies). The pressure measured was $30 \mathrm{kPa}$ peak positive. Based on these measurements, sensitivity is $240 \mathrm{mV} / \mathrm{MPa}$, with an NEP of $4.1 \mathrm{kPa}$ over $5-75 \mathrm{MHz}$ (this band covers the range of high-frequency imaging). The NEP of the present PROUD device is comparable to the state-of-the-art hydrophones of similar size. From the sensitivity, the optical resonance wavelength shift of the microring spectrum is approximately $200 \mathrm{pm} / \mathrm{MPa}$, and the dynamic range for ultrasound detection is about $700 \mathrm{kPa}$ peak-to-peak, adequate for imaging applications.

The pulse-echo (PE) waveform of the $20 \mathrm{MHz}$ transducer driven by an $8 \mathrm{~ns}$ pulse and its corresponding microring waveform were also recorded to determine the frequency response of the microring. The waveforms and their spectra are shown in Fig. 4. The PROUD device is sensitive over a range from 5 to $>40 \mathrm{MHz}$. Due to reflections of the pulse within the silicon substrate, a small ringing occurs following the waveform output by the microring. This can be removed by changing the substrate thickness so that the ringing is outside the frequency range of
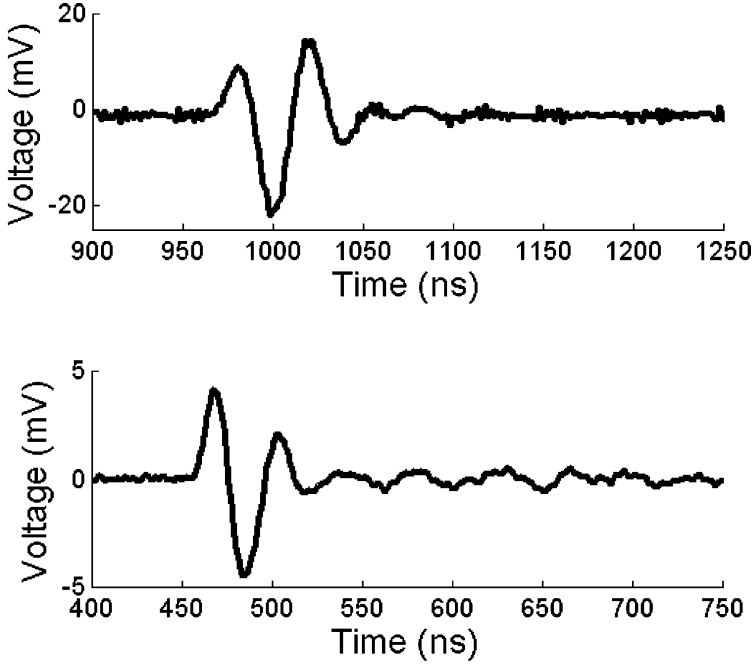

b)

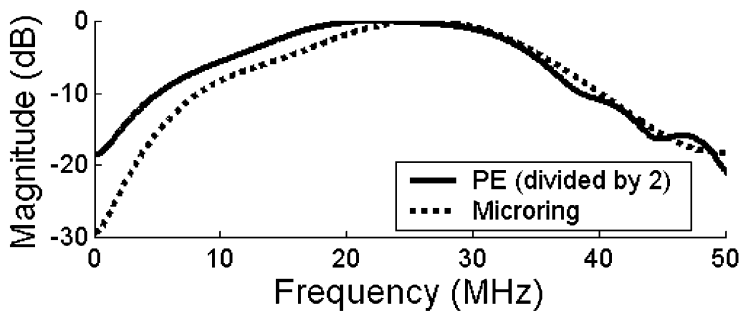

Fig. 4. Acoustic waveforms measured. (a) PE of the transducer. (b) Microring (c) Comparison of the spectra for both waveforms. The spectrum of the PE signal is divided by 2 to account for the two convolutions of the signal during PE measurements. Pulses are generated by a $20 \mathrm{MHz}$ unfocused transducer. Both signals were averaged 1000 times to produce the waveforms shown.

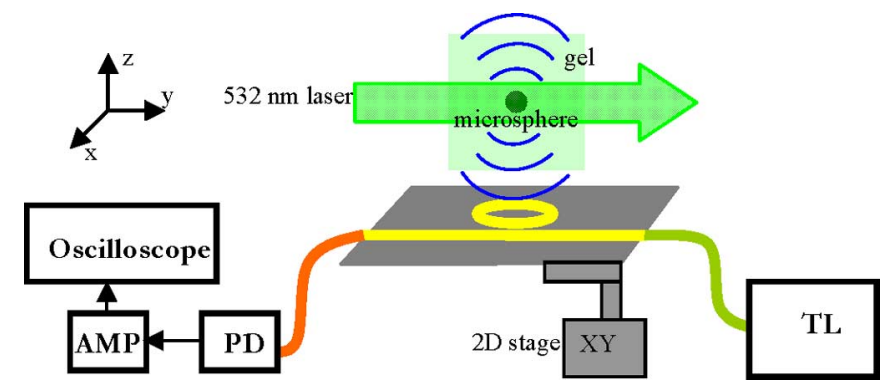

Fig. 5. Experimental apparatus for measuring angular sensitivity of the microring resonator and imaging of a $49 \mu \mathrm{m}$ microsphere. The PS microsphere produces a spherical wave, and the microring is scanned tangentially to the wave. The gel bulk and the microring were submerged in deionized water.

interest, or fabricating the microrings on a plastic substrate that has an acoustic impedance similar to water.

\section{Angular Response}

A photoacoustic method was used to test the angular dependence of sensitivity for the microring (see Fig. 5). A 49- $\mu$ mdiameter black PS microsphere (BK050, Microgenics Corp., Fremont, $\mathrm{CA}$ ) was set in a gel created by mixing $1.5 \%$ agarose (GPG/LE, American Bioanalytical, Natick, MA) with water. The microsphere was placed very close to the surface of the gel to minimize acoustic absorption. A $532 \mathrm{~nm}$ pulsed 

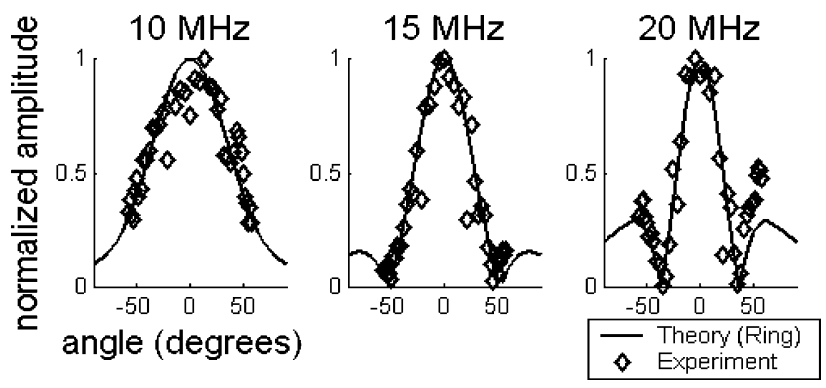

Fig. 6. Calculated and measured angular sensitivity of the microring at wavelengths of 10, 15, and $20 \mathrm{MHz}$. Experimental results agree well with the curves calculated using (2).

frequency-doubled Nd-YAG laser (Surelite I-20, Continuum, Santa Clara, CA) was used to illuminate the microsphere with a fluence of $112 \mathrm{~mJ} / \mathrm{cm}^{2}$. The bead's optical absorption at this wavelength causes transient heating and expansion of the sphere, and subsequently, generation of a spherical acoustic pulse.

The angular sensitivity for a single microring is shown in Fig. 6 for frequencies of 10, 15, and $20 \mathrm{MHz}$ signals. Signal levels at these frequencies were extracted by applying bandpass filters centered at the corresponding frequencies with $2 \mathrm{MHz}$ bandwidth to the recorded acoustic pulses, and then, detecting the peaks. The angular sensitivity shows frequency-dependant behavior. Such a frequency-dependent behavior can be understood by considering a ring piston transducer, where the theoretical angular response or directivity $D$, is given by [9]

$$
D(\theta)=J_{0}(k a \sin (\theta))
$$

where $k=\omega_{a} / c$ is the wavenumber of the incident acoustic signal, $a$ the average radius of the element, and $\theta$ the incident angle of the acoustic wave. The theoretical curve is also shown in Fig. 6, which fits well with the experimental data. For a given $k$, an element will generally have a wider angular sensitivity when the ring radius is reduced. One method of characterizing the angular response is to measure the $-6 \mathrm{~dB}$ beamwidth, or full-width at half maximum (FWHM). For beamforming applications, the beamwidth of the main lobe should extend to $+/-40^{\circ}$ incidence. For a $20 \mathrm{MHz}$ bandwidth, the element diameter must be less than $50 \mu \mathrm{m}$, and for $50 \mathrm{MHz}$ bandwidth, must be smaller than $25 \mu \mathrm{m}$ in order to comply with these requirements. For the microring detectors, smaller ring size can be made if the waveguide bending loss can be minimized, which can be achieved by operating at a shorter resonance wavelength, and by reducing the refractive index of the undercladding layer.

\section{IMAGING WITH PROUD}

\section{A. Photoacoustic Imaging}

Using the same setup for measuring the angular response, a 3-D photoacoustic image of the $49 \mu \mathrm{m}$ bead was obtained. The pulsed laser fluence was $10 \mathrm{~mJ} / \mathrm{cm}^{2}$. A 2-D translation stage, driven by two motorized linear actuators (T-LA60-A, Zaber Technologies Inc., Richmond, BC, Canada), scanned the microring to collect photoacoustic signals from the bead at different positions (each of which was an average of 4 records).

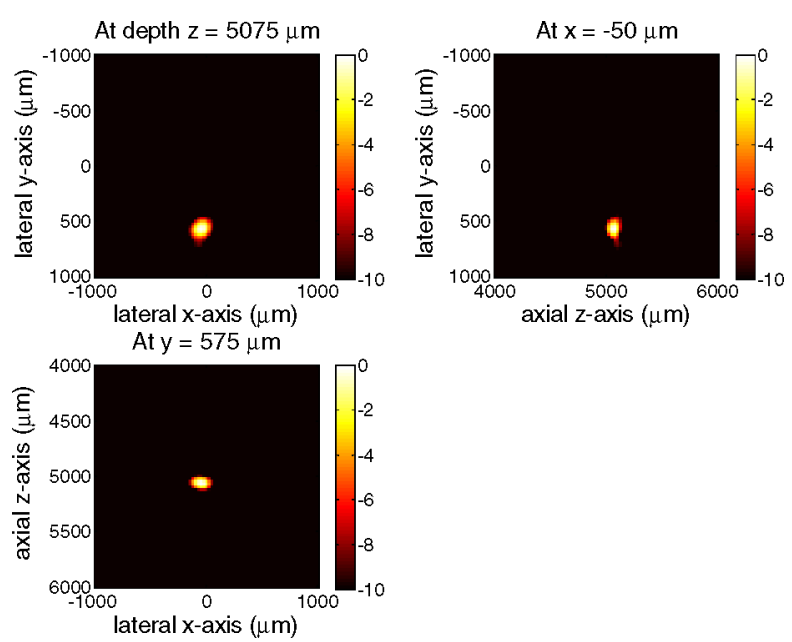

Fig. 7. 2-D cuts of the bead's 3-D image displayed in a $10 \mathrm{~dB}$ dynamic range. The bead diameter is $49 \mu \mathrm{m}$ but is imaged larger due to finite bandwidth and operating frequency limitations.
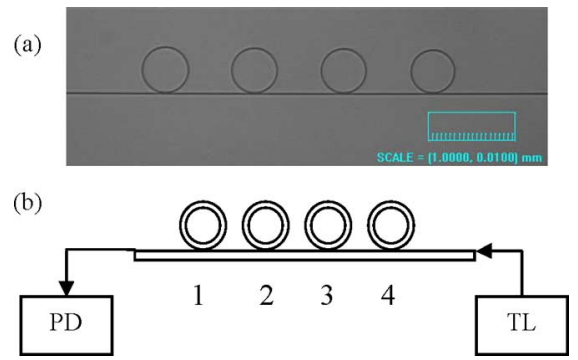

Fig. 8. Ring arrangement used for the mircoring array. (a) Optical micrograph of the fabricated four-ring device. (b) Four rings in serial create four distinct resonance peaks at the input to the photodetector. By using a wavelength tunable laser, each ring can be addressed individually.

Using this method, a $4.1 \mathrm{~mm}$ by $4.1 \mathrm{~mm}$ (virtual) 2-D array was formed at a $0.1 \mathrm{~mm}$ step. To reconstruct the image, a simple delay-and-sum beamforming algorithm was used, and a digital filter with a center frequency of $15 \mathrm{MHz}$ and a bandwidth of $16 \mathrm{MHz}$ was applied to the signals during beamforming. According to the array size and the bandwidth, the lateral and axial resolutions were around 150 and $90 \mu \mathrm{m}$, respectively. Fig. 7 shows three 2-D cuts of the 3-D image with a $10 \mathrm{~dB}$ dynamic range. The FWHM diameters of the reconstructed bead were 177, 184, and $108 \mu \mathrm{m}$ along the $x$ (approximately lateral), $y$ (approximately lateral), and $z$ (approximately axial) directions, respectively. These values are within reasonable range considering the bead's $49 \mu \mathrm{m}$ diameter and the resolutions of the imaging system. The images show that high-quality 3-D photoacoustic imaging is achievable using microrings on receive. With more rings working in parallel, frame rate can be increased.

\section{B. Four-Element Microring Array}

A microring array consisting of four microrings with a single input/output bus was also developed and tested (see Fig. 8). Each of the microrings is fabricated with a slightly different diameter (varied by $1.0 \mu \mathrm{m}$ per ring), resulting in a different resonant wavelength for each of the four rings. Therefore, 


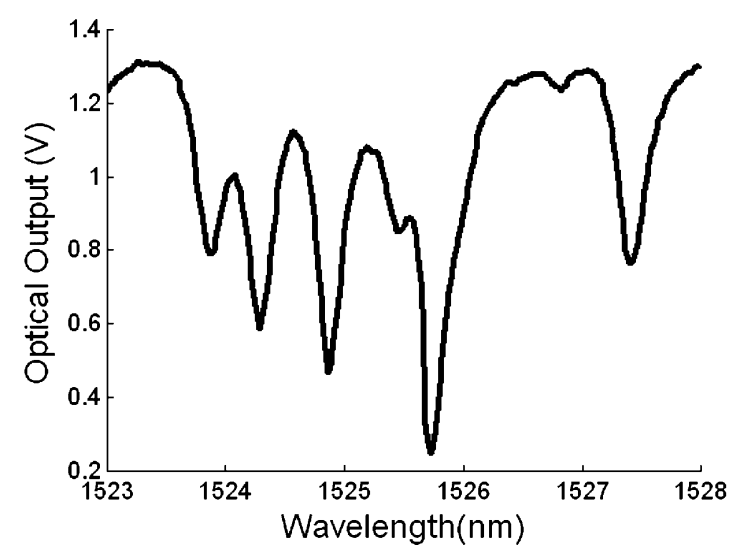

Fig. 9. Transmission spectrum for the microring array. Each resonance is labeled with the ring number to which it corresponds.

each ring element can be addressed through the same bus waveguide by tuning the input wavelength to match the resonance wavelength of that specific ring. A tunable laser source or multiwavelength diode lasers can be used to multiplex several wavelengths and address different elements. The ability to use different wavelengths of light to address different elements through the same bus waveguide greatly reduces the number of inputs and outputs, which makes this approach very attractive for in vivo ultrasound imaging applications.

The spectral response of the array is shown in Fig. 9. Peaks are labeled according to their element number. Note that more than four resonances are present. Two peaks may occur for one ring due to the presence of the TE and TM modes in the ring waveguide. This causes two shallower resonances rather than one deep resonance. Despite the slightly complex spectrum due to the existence of both modes, we were able to address each ring element without difficulty. By proper design of the waveguide cross-section dimension, we can eliminate the contribution of one of the modes, producing a cleaner spectrum for easy addressing of the ring elements.

In order to measure the spatial sensitivity of each element, a focused transducer with a small focal area is required. A $50 \mathrm{MHz} \mathrm{LiNbO}_{3}$ focused ultrasound transducer with aperture diameter $2.5 \mathrm{~mm}$, focal length $4 \mathrm{~mm}$ (Resource Center for Medical Ultrasonic Transducer Technology, University of Southern California, Los Angeles, CA) was used to insonify the microring array. The focal diameter of the transducer is approximately $50 \mu \mathrm{m}$. Four wavelengths were chosen corresponding to the resonant peak for each ring, and a 2-D scan with a $50 \mathrm{MHz}$ ultrasound transducer was performed over the entire array at each of the four wavelengths. The scanning step size was $25 \mu \mathrm{m}$. The waveform shape did not vary greatly between different elements although the elements with more shallow resonances gave lower peak sensitivities.

The spatial sensitivity for each of the four elements is shown in Fig. 10. The wavelengths used for generating the images were $1523.95,1524.38,1524.95$, and $1525.82 \mathrm{~nm}$, from top to bottom in order. A spatial scan at the resonance wavelength for the first element also displays sensitivity to element \#2. This is due to the overlap between the two elements' resonance curves.

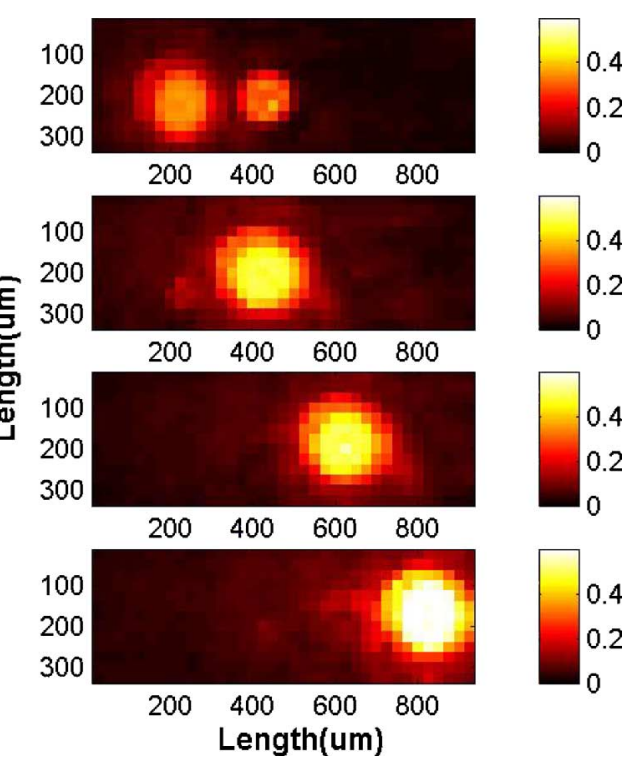

Fig. 10. 2-D spatial sensitivity of the microring array for each of the four wavelengths tested. Note the response of both the first and second ring to the first resonance wavelength $(1523.95 \mathrm{~nm})$. This is due to the overlap of the two resonance signals in the measured spectrum. Color indicates peak-to-peak voltage generated by the photodetector.

In addition, at $1527.53 \mathrm{~nm}$, only the first element is selected. The overlap in the ring resonances can be easily eliminated by increasing the $Q$ to make the resonances narrower and careful choice of the element sizes to obtain more equal resonance spacing. The active element diameter is measured to be approximately $125 \mu \mathrm{m}$. This is slightly larger than the actual element due to the $50-\mu \mathrm{m}$-focal area of the ultrasound transducer.

\section{DISCUSSION}

In this paper, we have demonstrated two aspects of the PROUD technology that are critical for employing it in highresolution imaging devices. The first is high sensitivity. A microring detector of $100 \mu \mathrm{m}$ size has an NEP of $4.1 \mathrm{kPa}$ in a bandwidth of $70 \mathrm{MHz}$. For comparison, the state-of-the-art piezoelectric PVDF transducer of similar size $(75 \mu \mathrm{m})$ has an NEP of $5.0 \mathrm{kPa}$ over the same bandwidth [10]. In addition, the PROUD device shows a broad angular response that accurately fits the expected response of a ring-shaped receiver. The second is the feasibility of array formation. As opposed to the electrical ultrasound transducers such as piezoelectric or capacitive, where individual coaxial transfer line is required for each element, a single optical waveguide can carry multiple signals for an array of PROUDs. This huge capacity is inherent to the use of optical information technology since the optical bandwidth is about $10^{5}$ times higher than the bandwidth of ultrasound signals (100 MHz). We have shown here how this principle can be realized to combine signals of four receivers on a common input/output waveguide. The scheme that we have used here can be further extended to larger number of elements in a linear array configuration and to 2-D arrays by using a cross configuration of input and output waveguides. 
While the potential benefits of the PROUD technology are highly attractive for the development of miniaturized ultrasound probes for high-resolution imaging, there are still significant challenges ahead. Reducing the size of the microring is essential for moving this technology toward applications. The benefit of it is twofold. From the acoustic aspect, it will result in higher operating frequency since the angular response can broaden and element density can increase. Typically, phased array ultrasound imaging probe requires an element size of half the acoustic wavelength. For example, to operate at a center frequency of $50 \mathrm{MHz}$, elements size should be reduced to $15 \mu \mathrm{m}$. Smaller rings would also increase the number of elements that can share a common waveguide since the free spectral range of each microring increases linearly with decreasing size. The major limitation on microring size reduction is optical loss due to high curvature (bending loss). To resolve this issue, we plan to design microrings operating in the visible range. Shorter wavelength and better optical properties of polymers in the visible range would allow forming high-quality resonators with size in the order of 10-20 $\mu \mathrm{m}$.

A second limitation of the current technique is the lack of ultrasound transmitter elements. Recently, our group presented significant improvements in thermoacoustic methods of laser ultrasound generation [11], [12]. These methods could be integrated on a planar optical chip with a microring receiver array to form a complete ultrasound imaging probe.

\section{CONCLUSION}

A new ultrasound sensor platform, PROUD, is presented. Polymers combine good optical properties and relatively high compressibility that make them an optimal choice for the PROUD. Experimentally, we have shown that the polymermicroring-based ultrasound receivers have high sensitivity, broad angular response, and high-frequency response. In addition, optical multiplexing allows a single waveguide to be used for probing a linear array of elements. We intend to further develop the technology for miniaturized high-frequency ultrasound imagers optimized for minimally invasive medical imaging applications. In principle, a complete linear array of 64 elements working at $50 \mathrm{MHz}$ can be designed to fit in a volume of $0.1 \times 0.1 \times 1 \mathrm{~mm}$. Such a device compaction would allow development of new clinical applications that will greatly enhance diagnostic capabilities of physicians. For example, in intravascular imaging, the imager could explore much smaller arteries and provide access to almost occluded sites. Another example is a "smart needle" where an imaging head is integrated into a small diameter biopsy needle. This would allow for on-site, real-time, high-resolution imaging. In many cases, this would form an alternative to biopsy. In others, it will guide the biopsy procedure for enhanced accuracy and sensitivity.

\section{REFERENCES}

[1] S. F. Foster, C. J. Pavlin, K. A. Harasiewicz, D. A. Christopher, and D. H. Turnbull, "Advances in ultrasound biomicroscopy," Ultrasound Med. Biol., vol. 26, pp. 1-27, 2000.

[2] B. E. Little, S. T. Chu, and H. A. Haus, "Second-order filtering and sensing with partially coupled traveling waves in a single resonator," Opt. Lett., vol. 23, pp. 1570-1572, 1998.
[3] C. Y. Chao, S. Ashkenazi, S.-W. Huang, M. O’Donnell, and L. J. Guo, "High-frequency ultrasound sensors using polymer microring resonators," IEEE Trans. Ultrason., Ferroelectr., Freq. Control, vol. 54, no. 5, pp. 957965, May 2007.

[4] C. Y. Chao, W. Fung, and L. J. Guo, "High Q-factor polymer microring resonators for biochemical sensing applications," IEEE Special Topics Quantum Electron., vol. 12, no. 1, pp. 134-142, Jan./Feb. 2006.

[5] C. Y. Chao and L. J. Guo, "Polymer micro-ring resonators fabricated by nanoimprint technique," J. Vac. Sci. Technol. B., vol. 20, no. 6, pp. 28622866, 2002.

[6] L. J. Guo, "Nanoimprint Lithography: Methods and material requirement," Adv. Mater., vol. 19, pp. 495-513, 2007.

[7] P. C. Beard, F. Perennes, and T. N. Mills, "Transduction mechanisms of the Fabry Perot polymer film sensing concept for wideband ultrasound detection," IEEE Trans. Ultrason., Ferroelectr., Freq. Control, vol. 46, no. 6, pp. $1575-1582$, Nov. 1999.

[8] A. D. Maxwell, O. A. Sapozhnikov, and M. R. Bailey, "A new PVDF membrane hydrophone for measurement of medical shock waves," presented at the IEEE 2006 Ultrason. Symp., Vancouver, BC, Canada, Oct., pp. 1608-1611.

[9] D. T. Blackstock, Fundamentals of Physical Acoustics. New York: Wiley, 2000.

[10] Precision Acoustics, Dorchester, U.K. Piezoelectric PVDF needle hydrophone of $75 \mu \mathrm{m}$ active detection size [Online]. Available: http://www. acoustics.co.uk/products/hpm04-1

[11] Y. Hou, J.-S. Kim, S. Ashkenazi, M. O’Donnell, and L. J. Guo, “Optical generation of high frequency ultrasound using two-dimensional gold nanostructure," Appl. Phys. Lett., vol. 89, pp. 93901-1-93901-3, 2006.

[12] Y. Hou, J.-S. Kim, S. Ashkenazi, S.-W. Huang, L. J. Guo, and M. O'Donnell, "Broadband all-optical ultrasound transducers," Appl. Phys. Lett., vol. 91, pp. 073507-1-073507-3, 2007.

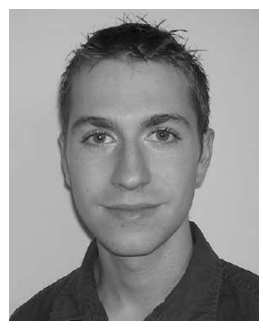

Adam Maxwell received the B.S. degree from the Department of Electrical Engineering, University of Washington, Seattle, in 2006.

From 2004 to 2006, he was with the Center for Industrial and Medical Ultrasound, University of Washington, where he was engaged in research on therapeutic ultrasound and lithotripsy. He is currently a Graduate Student in the Department of Electrical Engineering and Computer Science, University of Michigan, Ann Arbor. His current research interests include optoacoustic imaging, ultrasound dosimetry, and high-intensity focused ultrasound.

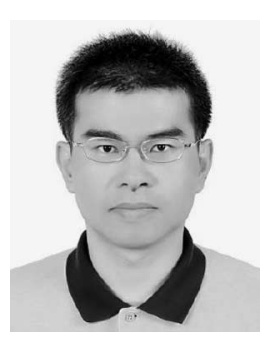

Sheng-Wen Huang (M'05) was born in Changhua, Taiwan, R.O.C, in 1971. He received the B.S. and Ph.D. degrees from the National Taiwan University, Taipei, Taiwan, in 1993 and 2004, respectively, both in electrical engineering.

From 2004 to 2005, he was as a Postdoctoral Researcher at the National Taiwan University. He is currently a Postdoctoral Researcher in the Department of Biomedical Engineering, University of Michigan, Ann Arbor. His current research interests include optoacoustic transduction and imaging, ultrasound elasticity imaging, and thermal strain imaging.
Tao Ling received the B.S. degree in physics from Nankai University, Tianjin, China, and the M.S. degree in optics from the Fudan University, Shanghai, China. He is currently working toward the Ph.D. degree in electrical engineering from the University of Michigan, Ann Arbor.

$\mathrm{He}$ is engaged in research on optical microcavity related biochemical sensor, imaging, and laser. 


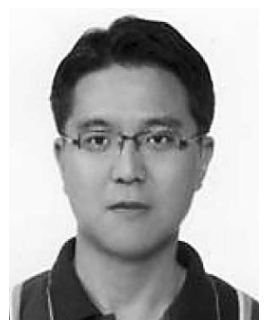

Jin-Sung Kim received the B.S. degree from the Department of Metallurgical Engineering, Hanyang University, Seoul, South Korea, in 1996, and the M.S. and Ph.D. degrees from the Department of Electrical Engineering and Computer Science, Syracuse University, Syracuse, NY, in 2000 and 2002, respectively.

He has been a Postdoctoral Researcher in the Electrical Engineering and Computer Science (EECS) Department, University of Michigan, Ann Arbor, where he was engaged in research in the field of nanotechnology. He is currently with LG Electronics Institute of Technology, Seoul, where he was for three years from 2002. His current research interests include optics and nanotechnology.

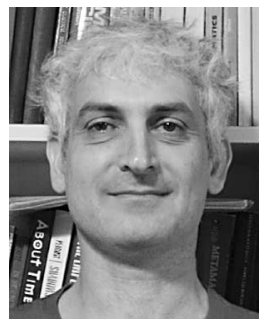

Shai Ashkenazi (M'00) received the B.Sc. degree in physics form the Technion Israel Institute of Technology, Haifa, Israel, in 1988, and the Ph.D. degree in physics from the Weizmann Institute of Science, Rehovot, Israel, in 1997.

He was engaged in the fields of ultrasonic and optical devices for medical applications in the research and development companies for six years. In 2003, he joined the University of Michigan, Ann Arbor, where he is currently a Research Scientist. His current research interests include medical imaging and nanotechnology.

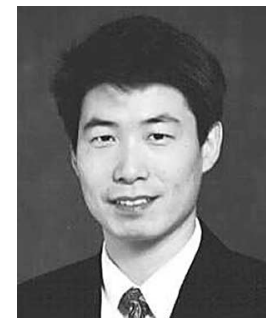

L. Jay Guo (S'96-M'97) received the Ph.D. degree from the University of Minnesota, Minneapolis, in 1997.

From 1998 to 1999, he was a Research Associate at Princeton University, Princeton, NJ. He is currently an Associate Professor in the Department of Electrical Engineering and Computer Science, University of Michigan, Ann Arbor. His current research interests include nanofabrication technologies with applications in polymer photonic devices, nanoimprint technology, organic electronics, and nanophotonics. 